\title{
Criadouros de Biomphalaria, temporários e permanentes, em Jaboatão dos Guararapes, PE
}

\author{
Temporary and permanent breeding sites for \\ Biomphalaria in Jaboatão dos Guararapes, PE
}

\author{
Marco Antônio Andrade de Souza ${ }^{1}$, Verônica Santos Barbosa', \\ Tereza Neuma Guedes Wanderlei ${ }^{2}$ e Constança Simões Barbosa ${ }^{1}$
}

\begin{abstract}
RESUMO
Realizou-se um inquérito malacológico em criadouros, permanentes e temporários, no bairro de Piedade, Jaboatão dos Guararapes, Pernambuco, entre novembro de 2006 e novembro de 2007 com o objetivo de conhecer a fauna malacológica dessa localidade, bem como o potencial de transmissão da esquistossomose mansoni. Além de Biomphalaria glabrata (Say, 1818), foram coletados moluscos Drepanotrema cimex (Moricand, 1837), Pomacea sp e Melanoides tuberculatus (Muller, 1774). Do total de Biomphalaria glabrata coletado, 1.490 exemplares encontraram-se vivos, sendo que 74 (5\%) estavam positivos para Schistosoma mansoni. 0 maior número de moluscos capturados e todos aqueles positivos para Schistosoma mansoni foram coletados na estação anual das chuvas. Foi observada também a presença de outras larvas de trematódeos infectando os moluscos Biomphalaria glabrata. Pertencentes às famílias Strigeidae e Diplostomatidae, apresentam, a primeira vista, morfologia que pode levar a confusão com as cercárias do Schistosoma mansoni, tornando-se indispensável seu conhecimento para o diagnóstico diferencial do agente causador da esquistossomose.
\end{abstract}

Palavras-chaves: Esquistossomose mansoni. Moluscos. Jaboatão dos Guararapes. Pernambuco.

\begin{abstract}
A malacological survey of permanent and temporary breeding sites was conducted in the Piedade neighborhood of Jaboatão dos Guararapes, Pernambuco, between November 2006 and November 2007, with the aim of determining the malacological fauna at this locality, along with the potential for Schistosomiasis mansoni transmission. In addition to Biomphalaria glabrata (Say, 1818), the molluscs Drepanotrema cimex (Moricand, 1837), Pomacea sp and Melanoides tuberculatus (Muller, 1774) were collected. Among the specimens of Biomphalaria glabrata that were collected, 1,490 were found alive, and 74 (5\%) were positive for Schistosoma mansoni. The largest numbers of molluscs collected, and all of the specimens that were positive for Schistosoma mansoni, were collected during the annual rainy season. The presence of larvae of other trematodes infecting the Biomphalaria glabrata molluscs was also observed. These trematodes were from the families Strigeidae and Diplostomatidae and, at first sight, they presented morphology that could lead to confusion with Schistosoma mansoni cercariae. Thus, knowledge of these trematodes becomes essential for the differential diagnosis of the etiological agent for schistosomiasis.
\end{abstract}

Key-words: Schistosomiasis mansoni. Molluscs. Jaboatão dos Guararapes. Pernambuco.

A humanidade convive com a esquistossomose desde a antiguidade, fato este comprovado por estudos que verificaram a presença de ovos de Schistosoma em múmias egípcias de 3.500a. C $^{8}$. Apesar de no Brasil a esquistossomose ter se estabelecido durante o período colonial, através do tráfico de escravos africanos parasitados ${ }^{1520}$, seu primeiro relato só foi descrito entre os anos de 1907 e 1908, por Pirajá da Silva, no Estado da Bahia, que supriu as incertezas taxonômicas quanto ao parasita, surgindo a partir de então as investigações sobre a distribuição geográfica e dados parasitológicos da doença ${ }^{2}$. Todavia, sua importância só foi evidenciada na década de 50 com a realização, por Pellon \& Teixeira, do grande inquérito

1. Parasitologia, Centro de Pesquisas Aggeu Magalhães, Fundação Oswaldo Cruz, Recife, PE. 2. Secretaria do Estado de Saúde de Pernambuco, Recife, PE. Apoio financeiro: CNPq - Conselho Nacional de Desenvolvimento Científico e Tecnológico.

Endereço para correspondência: Dr. Marco Antônio Andrade de Souza. Dept ${ }^{\circ}$ de Parasitologia/CPqAM/FIOCRUZ. Av. Moraes Rego s/n, Caixa Postal 7472 , Cidade Universitária, 50670-420 Recife, PE.

Tel: 5581 2101-2661.

e-mail: maandrades@cpqam.fiocruz.br

Recebido para publicação em: 26/03/2008

Aceito em: 11/06/2008 
coproscópico nacional de prevalência, inicialmente no nordeste do país e posteriormente em áreas supostamente não endêmicas do sul e sudeste. Desde então, houve um crescimento exponencial nas pesquisas sobre o Schistosoma mansoni e a doença por ele provocada $^{2817}$.

Estima-se que a esquistossomose afete entre 2.500.000 e 8.000.000 de indivíduos da população brasileira, sendo que no Estado de Pernambuco 15,2\% da população apresentam-se infectados ${ }^{5671217}$. De fato é endêmica nas Zonas da Mata e Agreste, estando distribuída em 72 dos 185 municípios do estado, cujas prevalências em algumas dessas localidades são superiores a $50 \%{ }^{10}{ }^{11}$. Contudo, desde o início dos anos 90, observa-se em Pernambuco o estabelecimento da esquistossomose em áreas litorâneas, quando foram registrados os primeiros casos de infecção pelo Schistosoma mansoni em veranistas e moradores da Praia de Itamaracá ${ }^{13}$, posteriormente em Porto de Galinhas ${ }^{4}$ e mais recentemente em Carne de Vaca, onde 17,2\% dos indivíduos analisados encontraram-se parasitados (CS Barbosa: dados não publicados). Em todos esses casos de infecção pelo Schistosoma mansoni, ocorridos em áreas litorâneas, os indivíduos infectados eram predominantemente de classes média e alta, apresentaram infecção aguda da doença e o molusco transmissor foi Biomphalaria glabrata. De certa forma, observa-se uma mudança no perfil epidemiológico da esquistossomose, que em áreas rurais incide principalmente na classe social de baixa renda, apresentase predominantemente na forma crônica e tem como vetor Biomphalaria stramine ${ }^{3}{ }^{4}$. Vale ressaltar a importância da Biomphalariaglabrata como transmissora, tanto por sua amplitude de distribuição, quanto pela eficiência na transmissão e da Biomphalaria straminea, encontrada em quase todas as bacias hidrográficas do Brasil, muito bem adaptada às variações climáticas ${ }^{22}$.

Desde 1999, ações do Programa de Controle da Esquistossomose (PCE) são realizadas no município de Jaboatão dos Guararapes. Porém, somente a partir de 2005 iniciou-se a alimentação dos bancos de dados do SIS/PCE. Neste ano, foram descritos relatos sobre a esquistossomose no Conjunto Habitacional Dom Hélder Câmara, no bairro de Piedade, verificando-se a presença de indivíduos parasitados por Schistosoma mansoni (TNG Wanderley: comunicação pessoal, 2006). Além disso, o encontro de Biomphalaria glabrata extremamente tolerante à variabilidade de concentrações salinas ${ }^{25}$, em coleções hídricas distantes apenas 500m da Praia de Piedade, motivou a realização deste trabalho, bem como de estudos epidemiológicos que permitam a observação dos indivíduos mais expostos ao Schistosoma mansoni nesses locais, facilitando assim a aplicação de tratamento adequado aos parasitados e posteriores medidas de controle da doença e dos moluscos transmissores.

\section{MATERIAL E MÉTODOS}

Características da área de estudo. Com uma população de 581.556 habitantes ${ }^{14}$, o município de Jaboatão dos Guararapes encontra-se na Região Metropolitana do Recife. Limita-se ao norte com Recife e São Lourenço da Mata, ao oeste com Moreno, ao Sul com Cabo de Santo Agostinho e ao leste com Oceano Atlântico.
Apresenta clima tropical quente e úmido, com temperatura média anual de $25,5^{\circ} \mathrm{C}$, atingindo média máxima de $26,6^{\circ} \mathrm{C}$ nos meses de janeiro e fevereiro e média mínima de $23,9^{\circ} \mathrm{C}$ em agosto. Dentre os corpos lagunares do município, está a Lagoa Olho D’ Água (anteriormente denominada Lagoa do Náutico), formada pelo afogamento dos rios que deságuam no litoral durante a última fase de avanço do mar (transgressão marinha) do período Holoceno. Bastante assoreada, esta lagoa sofre influência das oscilações das marés e das enchentes do Rio Jaboatão, além da poluição motivada por esgoto e lixo provenientes dos assentamentos localizados em seu entorno ${ }^{1}$, como o do conjunto habitacional Dom Hélder Câmara, que surgiu da necessidade de se abrigar moradores carentes do bairro de Piedade. No período de chuvas o acesso é dificultado pelos buracos formados nas vias e pela lama que invade as calçadas. Além isso, durante todo ano observa-se uma grande quantidade de galerias entupidas, que jorram o esgoto para a rua e para as coleções hídricas da região.

Dois criadouros temporários e um permanente, próximos à Lagoa Olho D' Água, distribuídos em uma área de aproximadamente $10.000 \mathrm{~m}^{2}$, nas intermediações de um campo de futebol coletivo, foram analisados nesse estudo. Ambos os criadouros temporários apresentaram área de $340 \mathrm{~m}^{2}$, aproximadamente e lâmina d' água cuja profundidade na cheia atinge $0,5 \mathrm{~m}$, ao passo que o criador permanente apresenta área de $1.200 \mathrm{~m}^{2}$, aproximadamente e lâmina d'água de 1,5m de profundidade durante o período das chuvas. Em todos os criadouros, foram observadas grandes quantidades de dejetos humanos provenientes das moradias das imediações.

Coleta de moluscos. Após escolha dos locais, os moluscos foram coletados utilizando-se de redes de mão (puçá), confeccionadas com nylon $(50 \mathrm{~cm}$ de largura, $40 \mathrm{~cm}$ de altura, $30 \mathrm{~cm}$ de abertura e $1 \mathrm{~mm}^{2}$ de malha), adaptadas a um cabo de madeira ou aço $(150 \mathrm{~cm}$ de comprimento). As coletas foram realizadas trimestralmente (1 dia de coleta), durante 1 ano, entre novembro de 2006 e novembro de 2007. Foi aplicado um esforço amostral individual de 30 minutos, por varredura, em cerca de $10 \mathrm{~m}$ de extensão, em cada um dos hábitats selecionados ${ }^{26}$. Todo o material coletado foi acondicionado em sacos plásticos, etiquetado e transportado para o Laboratório de Esquistossomose do Centro de Pesquisas Aggeu Magalhães, em Recife.

Exame de planorbídeos. No laboratório, após triagem manual, os moluscos foram contados, observados macroscopicamente e, a seguir, colocados individualmente em pequenos recipientes de vidro snap-cap, contendo $5 \mathrm{ml}$ de água isenta de cloro e deixados durante a noite para exame antes e após fotoestimulação artificial direta (lâmpada de 60W). No dia seguinte, após 2 horas de exposição à luz, o material foi examinado para verificação de emergência de cercárias de Schistosoma mansoni e de outros trematódeos. Os exames foram realizados semanalmente. Após 90 dias, aqueles moluscos que permaneceram negativos foram esmagados entre placas de vidro para a pesquisa de possíveis esporocistos e/ou metacercárias 9

De cada lote de planorbídeos coletados, retiraram-se cerca de $10 \%$ dos exemplares que foram sacrificados em água a $70^{\circ} \mathrm{C}$. As partes moles foram fixadas em líquido de Railliet-Henry e dissecadas sob microscópio estereomicroscópio ${ }^{24}$. Para a 
identificação das espécies, foram considerados os parâmetros conquiliológicos e morfológicos, segundo diversas fontes bibliográficas $^{182} 23$.

Inquérito coproscópico. Amostras de fezes de 446 moradores do Conjunto Habitacional Dom Hélder Câmara foram coletadas e examinadas pelo método quantitativo de Kato Kat ${ }^{16}$ (2 lâminas) no laboratório de análises clínicas, da regional de saúde, do município de Jaboatão dos Guararapes, durante o ano de 2006.

\section{RESULTADOS}

Coleta de moluscos. Foram coletados 1.635 moluscos Biomphalaria glabrata em três estações de coleta ( 2 criadouros temporários e 1 permanente), previamente estabelecidas, próximas à Lagoa Olho D’Água, nas cercanias de um campo de futebol coletivo (Figuras 1 a 3). Do total coletado, 1.490 exemplares encontraram-se vivos, sendo que 74 (5\%) estavam positivos para Schistosoma mansoni. Foi observada também a presença de outras larvas de trematódeos de cauda bifurcada, pertencentes às famílias Strigeidae e Diplostomatidae, infectando

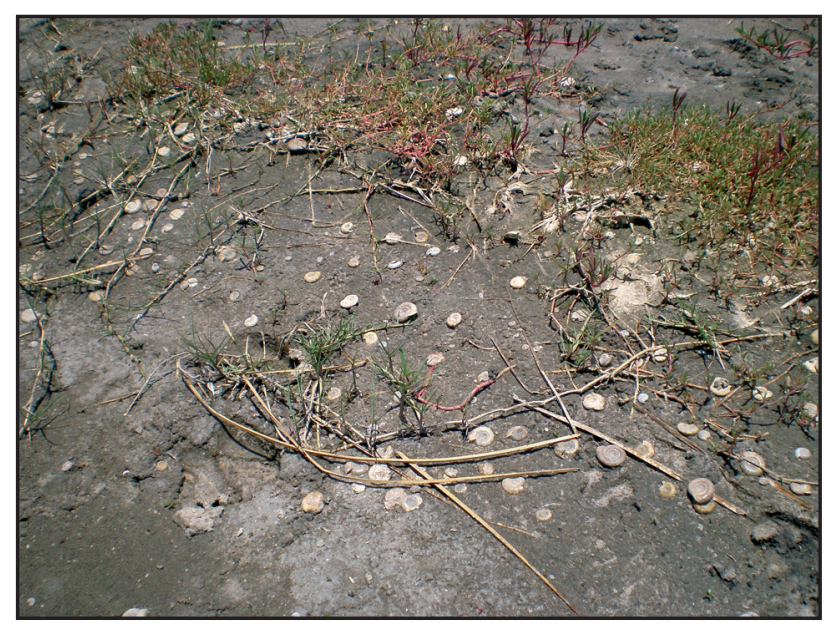

Figura 1 - Estação de coleta 1 (S $08^{\circ} 11$ '30.1” W $\left.034^{\circ} 56^{\prime} 23.0^{\prime \prime}\right)$ Criadouro temporário - Bairro de Piedade, Jaboatão dos Guararapes, $\boldsymbol{P E}$.

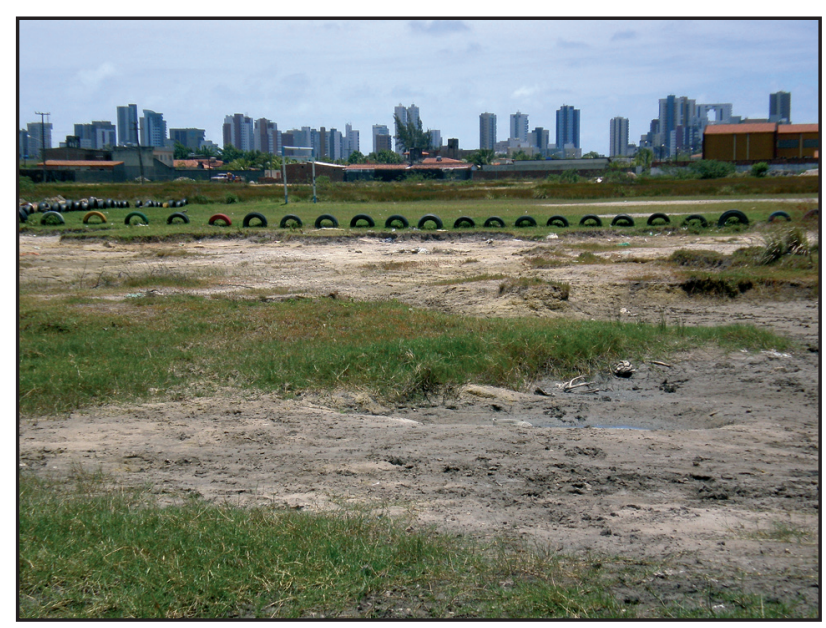

Figura 2 - Estação de coleta 2 (S $08^{\circ} 11$ ' 25.9” W $034^{\circ} 56^{\prime} 21.4$ ") Criadouro temporário - Bairro de Piedade, Jaboatão dos Guararapes, PE.

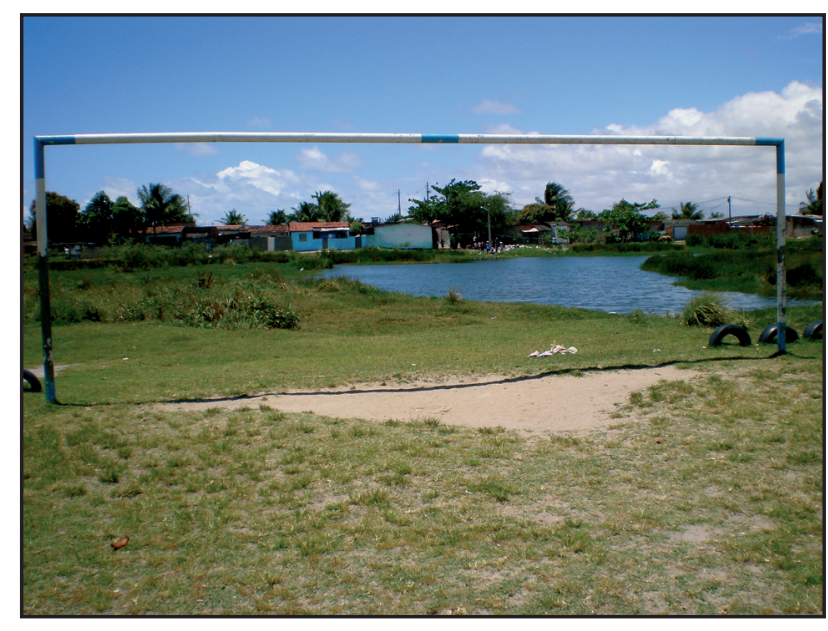

Figura 3 - Estação de coleta 3 ( $S$ 08 ${ }^{\circ} 11$ ' 29.3" W $034^{\circ} 56^{\prime} 18.1$ ”) Criadouro permanente - Bairro de Piedade, Jaboatão dos Guararapes, Pernambuco.

os moluscos Biomphalaria glabrata. Do total de moluscos vivos coletados, $50(3,4 \%)$ exemplares estavam infectados por esses helmintos.

0 número de moluscos coletados foi significativamente menor na $1^{\text {a }}, 2^{\text {a }}$ e $5^{\text {a }}$ fases de coleta, realizadas em novembro de 2006, fevereiro e novembro de 2007 (Tabela 1), períodos do ano considerados de seca. Além do molusco transmissor da esquistossomose, foram coletados ainda 179 exemplares de Drepanotrema cimex, 9 Pomacea sp e 12 Melanoides tuberculatus, encontrados apenas durante os meses de maio e agosto de 2007 (Tabela 2).

Coproscopia. 0 exame parasitológico de fezes foi realizado em 446 indivíduos, durante 0 ano de 2006, e detectou a presença de ovos de Schistosoma mansoni em 94 deles, representando um índice de infecção de 21,1\%, considerado o maior do município. Para quantificar a intensidade de infecção, os indivíduos foram

Tabela 1- Moluscos Biomphalaria glabrata coletados no Bairro de Piedade, Jaboatão dos Guararapes, Pernambuco, entre novembro de 2006 e novembro de 2007.

\begin{tabular}{|c|c|c|c|c|}
\hline Períodos de coleta & Estações de coleta & Vivos & Mortos & $\begin{array}{c}\text { Positivos para } \\
\text { Schistosoma mansoni }\end{array}$ \\
\hline & Estação 1 & 8 & 12 & 0 \\
\hline \multirow[t]{3}{*}{ Novembro de 2006} & Estação 2 & 7 & 5 & 0 \\
\hline & Estação 3 & 6 & 8 & 0 \\
\hline & Estação 1 & 13 & 14 & 0 \\
\hline \multirow[t]{3}{*}{ Fevereiro de 2007} & Estação 2 & 6 & 5 & 0 \\
\hline & Estação 3 & 8 & 4 & 0 \\
\hline & Estação 1 & 334 & 16 & 16 \\
\hline \multirow[t]{3}{*}{ Maio de 2007} & Estação 2 & 204 & 8 & 8 \\
\hline & Estação 3 & 308 & 10 & 12 \\
\hline & Estação 1 & 276 & 14 & 15 \\
\hline \multirow[t]{3}{*}{ Agosto de 2007} & Estação 2 & 139 & 9 & 9 \\
\hline & Estação 3 & 160 & 15 & 14 \\
\hline & Estação 1 & 8 & 10 & 0 \\
\hline \multirow[t]{2}{*}{ Novembro de 2007} & Estação 2 & 6 & 9 & 0 \\
\hline & Estação 3 & 7 & 6 & 0 \\
\hline Total & & 1.490 & 145 & 74 \\
\hline
\end{tabular}


Tabela 2 - Número e outras espécies de moluscos coletados no Bairro de Piedade, Jaboatão dos Guararapes, Pernambuco, nos meses de maio e agosto de 2007.

\begin{tabular}{ccccc}
\hline & \multicolumn{4}{c}{ Espécies de moluscos } \\
\cline { 2 - 5 } Período de coleta & $\begin{array}{c}\text { Drepanotrema } \\
\text { cimex }\end{array}$ & Pomacea sp & $\begin{array}{c}\text { Melanoides } \\
\text { tuberculatus }\end{array}$ & Total \\
\hline Maio de 2007 & 10 & 0 & 1 & 11 \\
Estação de coleta 1 & 13 & 1 & 2 & 16 \\
Estação de coleta 2 & 52 & 3 & 3 & 58 \\
Estação de coleta 3 & & & & \\
Agosto de 2007 & 11 & 1 & 1 & 13 \\
Estação de coleta 1 & 20 & 1 & 0 & 21 \\
Estação de coleta 2 & 73 & 3 & 5 & 81 \\
Estação de coleta 3 & 179 & 9 & 12 & 200 \\
\hline Total & & & & \\
\hline
\end{tabular}

classificados em três categorias: apresentando entre 1 e 4 ovos por grama de fezes $(\mathrm{OPG})$, entre 5 e $16 \mathrm{OPG}$ e 17 ou mais $\mathrm{OPG}$, considerados de baixa, média e alta parasitemias, respectivamente. Foram verificados 43 indivíduos apresentando entre um e quatro $\mathrm{OPG}, 36$ apresentando entre 5 e 16 OPG e outros 15 apresentando 17 ou mais ovos por grama de fezes. Vale ressaltar que apesar das ações do programa de controle da esquistossomose terem sido implantadas em Jaboatão dos Guararapes em 1999 e a alimentação do banco de dados do SIS/PCE realizada a partir de 2005, neste mesmo ano e em 2007, não foi trabalhada a comunidade de Dom Hélder.

\section{DISCUSSÃO}

O início das chuvas de verão na região metropolitana de Recife significa transtornos e prejuízos aos moradores que vivem nas proximidades da Lagoa Olho D' Água. São dezenas de bairros afetados pelas enchentes constantes, tanto em regiões mais nobres, próximas a orla, como nas comunidades mais carentes. 0 conjunto habitacional Dom Hélder Câmara, localizado em Piedade é só um exemplo do transtorno que poucos minutos de chuva podem provocar naquela região. As ruas viram verdadeiros rios de água suja, alagam residências e estabelecimentos comerciais, dificultando o deslocamento dos moradores.

Além dos problemas inerentes às chuvas torrenciais, tais como o grande número de buracos nas vias públicas, congestionamentos, proliferação de doenças como hepatite e leptospirose há o acúmulo de água nos criadouros temporários, que durante boa parte do ano permanecem secos, acarretando em uma explosão demográfica dos moluscos estivados. Em particular, Biomphalaria glabrata, única espécie vetora da esquistossomose mansoni na localidade estudada, o que reforça sua importância epidemiológica como transmissora da doença no litoral pernambucano ${ }^{4}$.

Apesar de terem sido encontrados em todas as coletas realizadas durante o período de estudos, verificou-se que o maior número de exemplares de Biomphalaria glabrata capturados foi na estação das chuvas, assim como todos os moluscos positivos para Schistosoma mansoni.
Vale ressaltar que o clima tropical do país, especialmente em Pernambuco, associado à enorme variedade de hábitats aquáticos, as altas temperaturas e a luminosidade intensa contribuem para propagação de vetores da esquistossomose e a manutenção da doença, principalmente pela falta de educação sanitária e de saneamento público. Estes são fatores fundamentais para 0 estabelecimento de focos de transmissão, proporcionando a contaminação dos locais de criadouros de moluscos suscetíveis por fezes contendo ovos viáveis de Schistosoma mansoni ${ }^{19}$. Em especial, nas proximidades das estações de coletas previamente estabelecidas, uma grande quantidade de lixo residencial e industrial e de dejetos humanos foi observada. Não obstante, esses locais, por estarem próximos a um campo de futebol coletivo, são amplamente utilizados pela comunidade em suas atividades de lazer, o que reflete nos índices de infecção humana, cujas cargas parasitárias foram bastante significativas e de moluscos transmissores observados. Além disso, a partir da constatação de que populações de Biomphalaria glabrata resistem a valores ambientais físico-químicos bem acima dos referenciados na literatura ${ }^{25}$, apresentando extrema tolerância à variabilidade de concentrações salinas nas águas e nos sedimentos e pela proximidade dos locais de coleta às praias de Jaboatão dos Guararapes, é salutar recomendar aos órgãos de vigilância em saúde ambiental o monitoramento e maior atenção às coleções hídricas salobras, especialmente em regiões endêmicas para a esquistossomose. Não bastasse isso, o encontro de outros tipos de larvas de trematódeos infectando os moluscos do gênero Biomphalaria e que apresentam morfologia que em um primeiro momento de observação podem levar a confusão com as cercárias do Schistosoma mansoni, reforça a necessidade do aprimoramento dos conhecimentos para o diagnóstico diferencial do agente causador da esquistossomose.

\section{AGRADECIMENTOS}

Aos técnicos da Fundação Nacional de Saúde, Sebastião Alves dos Santos e do Laboratório do Serviço de Referência em Esquistossomose do Centro de Pesquisas Aggeu Magalhães, Valdeci Francisco de Oliveira, pelo apoio incondicional durante as realizações dos trabalhos de campo.

\section{REFERÊNCIAS}

1. Agência Estadual do Meio Ambiente e Recursos Hídricos - CPRH. Diagnóstico do Meio Físico e Biótico e Mapa do Uso e Ocupação do Solo do Núcleo Metropolitano do Litoral Pernambucano. Recife, 2006. Disponível em: http://www.cprh.pe.gov. br. Acesso em 07 de fevereiro de 2008.

2. Andrade ZA. A esquistossomose no Brasil após quase um século de pesquisas. Revista da Sociedade Brasileira de Medicina Tropical 35: 509-513, 2002.

3. Araújo KCGM, Resendes APC, Souza-Santos R, Silveira-Júnior JC, Barbosa CS. Análise espacial dos focos de Biomphalaria glabrata e de casos humanos de esquistossomose mansônica em Porto de Galinhas, Pernambuco, Brasil, no ano 2000. Cadernos de Saúde Pública 23: 409-417 2007.

4. Barbosa CS, Domingues ALC, Abath F, Montenegro SML, Guida U, Carneiro J, Tabosa B, Morais CNL, Spinelli V. Epidemia de esquistossomose aguda na praia de Porto de Galinhas, Pernambuco, Brasil. Cadernos de Saúde Pública 17: 725-728, 2001. 
5. Barbosa CS, Silva CB, Barbosa FS. Esquistossomose: reprodução e expansão da endemia no Estado de Pernambuco no Brasil. Revista de Saúde Pública 30: 609-616, 1996

6. Burlandy-Soares LC, Dias LCS, Kanamura HY, Oliveira EJ, Ciaravolo RM Schistosomiasis mansoni: follow up of control program based on parasitologic and serologic methods in a brazilian community of low endemic. Memórias do Instituto Oswaldo Cruz 98: 853-859, 2003.

7. Carvalho EMF, Acioli MD, Branco MAF, Costa AM, Cesse EAP, Andrade AG, Mello EMLL. Evolução da esquistossomose na Zona da Mata Sul de Pernambuco. Epidemiologia e situação atual: controle ou descontrole? Cadernos de Saúde Pública 14: 787-795, 1998.

8. Coura JR, Amaral RS. Epidemiological and control aspects of schistosomiasis in brazilian endemic areas. Memórias do Instituto Oswaldo Cruz 9 (supl I): 13-19, 2004

9. Coutinho J0. Índices de infestação natural dos planorbídeos pelas cercárias do Schistosoma mansoni na cidade do Salvador-Bahia. Anais da Faculdade de Medicina de São Paulo 25: 29-53, 1950.

10. Farias LMM, Resendes APC, Sabrosa PC, Souza-Santos R. Análise preliminar do sistema de informação do Programa de Controle da Esquistossomose no período de 1999 a 2003. Cadernos de Saúde Pública 23: 235-239, 2007.

11. Favre TC, Pieri OS, Barbosa CS, Beck L. Avaliação das ações de controle da esquistossomose implementadas entre 1977 e 1996 na área endêmica de Pernambuco, Brasil. Revista da Sociedade Brasileira de Medicina Tropical 34: 569-576, 2001.

12. Gazzinelli MF, Gazzinelli A, Santos RV, Gonçalves LAO. A interdição da doença: uma construção cultural da esquistossomose em área endêmica, Minas Gerais, Brasil. Cadernos de Saúde Pública 18: 1629-1638, 2002.

13. Gonçalves JF, Santana W, Barbosa CS, Coutinho A. Esquistossomose aguda, de caráter episódico, na Ilha de Itamaracá, Estado de Pernambuco. Cadernos de Saúde Pública 7: 424-425, 1991.

14. Instituto Brasileiro de Geografia e Estatística. Censo 2000. Disponível em: http://www.ibge.gov.br/censo/. Acesso em 07 de fevereiro de 2008.
15. Katz N. Brazilian contributions to epidemiological aspects of schistosomiasis mansoni. Memórias do Instituto 0swaldo Cruz 87 (supl IV): 1-9, 1992.

16. Katz N, Chaves A, Pellegrino J. A simple device for quantitative stool thick smear technique in schistosomiasis mansoni. Revista do Instituto de Medicina Tropical de São Paulo 14: 397-400, 1972.

17. Katz N, Peixoto SV. Análise crítica da estimativa do número de portadores de esquistossomose mansoni no Brasil. Revista da Sociedade Brasileira de Medicina Tropical 33: 303-308, 2000.

18. Malek EA. Laboratory guide and notes for medical malacology. Minneapolis, Burgess Publishing Company, 1962.

19. Melo AL, Coelho PMZ. Schistosoma mansoni. In: Neves DP, Melo AL, Genaro O, Linardi PM (eds) Parasitologia Humana, 10ª edição, Editora Atheneu, São Paulo, p. $174-193,2000$

20. Morgan JAT, Dejong RJ, Snyder SD, Mkoji GM, Loker ES. Schistosoma mansoni and Biomphalaria: past history and future trends. Parasitology 123: 211-228, 2001.

21. Pan American Health Organization. A guide for the identification of the snail intermediate hosts of schistosomiasis in the Americas. Scientific Publication 168, 1968.

22. Paraense WL. Fauna planorbídica do Brasil. In: Lacaz CS, Baruzzi GR, Siqueira JRW (eds) Introdução à geografia médica do Brasil. Universidade de São Paulo, p. 213-239, 1972.

23. Paraense WL. Estado atual da sistemática dos planorbídeos brasileiros. Arquivos do Museu Nacional do Rio de Janeiro 55: 105-128, 1975.

24. Paraense WL, Deslandes N. Observations on the morphology of Australorbis nigricans. Memórias do Instituto Oswaldo Cruz 53: 121-124, 1955.

25. Silva $\mathrm{PB}$, Barbosa CS. Aspectos físico-químicos e biológicos relacionados à ocorrência de Biomphalaria glabrata em focos litorâneos da esquistossomose em Pernambuco. Química Nova 29: 901-906, 2006.

26. Souza MAA, Souza LA, Machado-Coelho GLL, Melo AL. Levantamento malacológico e mapeamento das áreas de risco para transmissão da esquistossomose mansoni no município de Mariana, Minas Gerais, Brasil. Revista de Ciências Médicas e Biológicas 5: 132-139, 2006 